\title{
Predictive criteria for MRI-based evaluation of response both during and after radiotherapy for cervical cancer
}

\author{
Jordy Mongula, MD', Brigitte Slangen, MD, PhD'.2, Doenja Lambregts, MD³. Frans Bakers, MD³, Shekar Mahesh, MD³, \\ Ludy Lutgens, MD, PhD2,4, Toon Van Gorp, MD, PhD 1,2, Roy Vliegen, MD, PhD5 , Prof. Roy Kruitwagen, MD, PhD',2, \\ Prof. Regina Beets-Tan, MD, PhD2,3 \\ 'Department of Obstetrics \& Gynecology, Maastricht University Medical Centre, Maastricht, ${ }^{2}$ GROW. School for Oncology and Developmental \\ Biology, Maastricht, ${ }^{3}$ Department of Radiology, Maastricht University Medical Centre, ${ }^{4}$ MAASTRO Clinic, Maastricht, ${ }^{5}$ Department of \\ Radiology, Heerlen Atrium Medical Centre, Heerlen, The Netherlands
}

\begin{abstract}
Purpose: For cervical carcinoma, the presence of persistent disease after radiotherapy (RT) is a significant predictor for survival. To date, no standard protocol is available to evaluate a response. This study was performed to assess magnetic resonance imaging (MRI) to evaluate presence of local residual disease during and after RT for Federation of Gynecology and Obstetrics (FIGO) stage Ib1-IVa cervical cancer.

Material and methods: Forty-two patients were included. Patients underwent MRI before external beam RT, at final intracavitary brachytherapy (BCT) and 2-3 months after completion of RT. Two blinded radiologists (observer 1: experienced, observer 2: less experienced) scored the likelihood of residual tumor. Magnetic resonance imaging was evaluated by means of (a) 'subjective' visual evaluation of T2 weighted MRI images, and (b) 'objective' visual evaluation of T2 weighted MRI images according to predefined imaging criteria.

Results: Seven patients had residual disease. Area under the receiver operating characteristics curve (AUC) for 'subjective' visual assessment was $0.79 / 0.75$ (observer 1/observer 2) after RT and 0.75/0.43 at final BCT. The combined 'objective' MRI criteria (isointense, nodular, and irregular) resulted in improved prediction of residual tumor (AUCs of 0.91/0.85 after RT). For the less experienced observer, the MRI criteria set significantly improved prediction of residual tumor compared to 'subjective' visual assessment. Observer dependency decreased, kappa of 0.41 compared to 0.84 for the MRI criteria set after RT.

Conclusion: Compared to 'subjective' visual assessment, predefined 'objective' MRI criteria increase diagnostic performance and decrease observer dependency for assessing residual tumor after RT in cervical cancer.

Key words: brachytherapy, cervical carcinoma, cervical cancer, magnetic resonance imaging, radiation therapy.

Purpose

Cervical cancer is the third most common cancer worldwide and has an annual mortality rate of 270,000. Approximately $30 \%$ of patients die due to persistent or recurrent disease after therapy. Recurrence mainly occurs during the first 2 years after treatment $(60-90 \%$ of cases) $[1,2,3]$. The presence of persistent disease after radiotherapy (RT) is a significant predictor of patient survival. Although surgery has been suggested as an adjuvant therapy for cervical carcinoma patients after RT, it is clear that it does not seem to improve overall survival [4,5]. Moreover, the addition of salvage surgery to RT may increase both morbidity and cost. Therefore, salvage sur-

gery should be reserved for selected cases with residual tumor $[6,7]$. To allow for such treatment stratification, it is necessary to accurately evaluate tumor responses after RT. However, no standard protocol for evaluating tumor response after RT is available. Gynecologic examination combined with biopsies several weeks after RT is often performed routinely. Though, it is difficult to recognize small tumors within areas of radiation-induced fibrosis in suspicious cases [4]. Furthermore, sampling errors may explain why, even with random biopsies, small microscopic tumor sites are easily missed, especially when suspected lesions are located outside the cervix or vagina. More accurate and (preferably) less invasive diagnostic modalities are required to allow a more reliable identifica-
\end{abstract}


tion of patients who require salvage surgery to improve survival.

Magnetic resonance imaging (MRI) is often used to assess responses of various tumors to RT including pelvic and cervical tumors $[8,9,10,11,12]$. However, it is unclear which MR criteria are diagnostic for residual tumor tissue after RT. After treatment, the irradiated tumor bed is often replaced by fibrotic tissue. The problem with MRI is that differentiating fibrotic tissue from residual tumor tissue is difficult; for example, MRI of rectal cancer results in a false-positive rate of $50 \%[8,13]$. The aims of the present study were to evaluate the ability of pelvic MRI to detect residual tumor after RT, to identify and validate objective imaging criteria predictive for residual tumor, and to assess their performance.

\section{Material and methods}

\section{Patients}

Data for 42 patients with primary cervical cancer (International Federation of Gynecology and Obstetrics

Table 1. Baseline characteristics, patients $(n=42)$

\begin{tabular}{|c|c|}
\hline \multicolumn{2}{|l|}{ Age } \\
\hline Median (range) & 53 years $(31-81)$ \\
\hline \multicolumn{2}{|l|}{ Histology } \\
\hline Squamous cell carcinoma & $33(79 \%)$ \\
\hline Adenocarcinoma & $7(17 \%)$ \\
\hline Adenosquamous carcinoma & $2(4 \%)$ \\
\hline \multicolumn{2}{|l|}{ FIGO } \\
\hline \multicolumn{2}{|l|}{ । } \\
\hline lb1 & $2(5 \%)$ \\
\hline lb2 & $6(14 \%)$ \\
\hline \multicolumn{2}{|l|}{ II } \\
\hline$\| \mathrm{Ia}$ & $8(19 \%)$ \\
\hline $\mathrm{Ilb}$ & $17(40 \%)$ \\
\hline \multicolumn{2}{|l|}{ III } \\
\hline IIIa & $1(2 \%)$ \\
\hline $\mathrm{IIlb}$ & $4(10 \%)$ \\
\hline \multicolumn{2}{|l|}{ IV } \\
\hline IVa & $4(10 \%)$ \\
\hline \multicolumn{2}{|l|}{ Therapy } \\
\hline Radiotherapy & $2(5 \%)$ \\
\hline Chemo-radiotherapy & $28(67 \%)$ \\
\hline Hyperthermia-radiotherapy & $12(28 \%)$ \\
\hline \multicolumn{2}{|c|}{ Time between last radiotherapy and MRI } \\
\hline Median (range) & 9 weeks (4-51) \\
\hline \multicolumn{2}{|l|}{ Follow-up } \\
\hline Median (range) & 24 months (13-81) \\
\hline
\end{tabular}

FIGO - International Federation of Gynecology and Obstetric, MRI - magnetic resonance imaging
[FIGO] stage $\geq \mathrm{Ib} 1$ ) who were referred to our gynecologic oncological center (Maastricht University Medical Centre, Maastricht, The Netherlands) for radiation therapy between May 2004 and August 2010 were analyzed retrospectively. The inclusion criteria were as follows: (a) histologically-proven primary cervical carcinoma, (b) availability of pre- and post-treatment pelvic MRIs, (c) treatment with external beam radiation therapy (EBRT) (46.0-50.4 Gy), high-dose-rate brachytherapy with chemotherapy (BCT) or without chemotherapy (CT), or hyperthermia (HT). Initially, BCT comprised 2-3 fractions for prescribing a total dose between 17-21 Gy. However, this has been replaced with MR-image-guided intra-cavitary plus interstitial BCT, which comprises 3-4 fractions of 7 Gy according to GEC-ESTRO guidelines. The BCT was delivered over two or three sessions, with a 1-week interval between each session. The first BCT session was usually scheduled during the $5^{\text {th }}$ week of EBRT. Typically, the overall treatment time was 6-7 weeks.

The baseline characteristics of the patients are listed in Table 1. Patients with distant metastasis at the time of presentation were excluded. The retrospective nature of the study meant that ethics board approval was not required (not subject to the Medical Research Involving Human Subjects Act).

\section{Magnetic resonance imaging}

The majority of the MRI examinations (64\%) were performed using a 1.5-Tesla MRI unit (Intera Achieve; Philips Medical Systems, Best, The Netherlands; or Siemens Magnetom Avanto, Erlangen, Germany) and with a phased array surface coil. Patients were placed in a feetfirst supine position. The imaging protocol comprised standard 2-dimensional $\mathrm{T} 2 \mathrm{~W}$ fast spin-echo images in three orthogonal directions (TR/TE (3200-3427)/(100$150 \mathrm{msec}), 90-150^{\circ}$ flip angle, 18-24 echo train length, 3-4 NSA, $0.98 \times 0.98 \times(3.00-5.00) \mathrm{mm}$ acquisition voxel size, 24-48 slices, 3.15-5.33 min acquisition time). The axial and coronal images were angled perpendicular and parallel to the cervical axis, respectively. The remaining MR examinations $(36 \% ; 44 \%$ of which comprised examinations performed during BCT) were performed using a 3.0-Tesla MRI unit using a similar protocol (T2W FSE; TR/TE [7000/150], 28 echo train length, 2 NSA, $0.98 \times 0.98 \times$ (3.00-4.00) $\mathrm{mm}$ acquisition voxel size, 40 slices, $3.15 \mathrm{~min}$ acquisition time). Patients received neither bowel preparation nor anti-spasmodic agents during the MRI examinations. Magnetic resonance imaging was performed at different time points: (a) before the onset of external beam radiation treatment, (b) immediately before the final BCT application, and (c) at a median 9 weeks (range 4-51 weeks) after the completion of radiotherapy.

\section{Image evaluation}

The MR images were independently scored by a senior radiologist (FB, reader 1 ) and a junior radiologist (SM, reader 2), who have been 7 and 2 years experienced in pelvic MR imaging, respectively. The two readers were only aware of the initial FIGO stage but were blinded to each other's interpretation of the results and the fol- 
low-up data. First, the readers were asked to assess the presence of residual tumor based on a 'subjective' visual assessment of the $\mathrm{T} 2 \mathrm{~W}$ images using the following confidence level scores: 0 - definitely no residual tumor, 1 - probably no residual tumor, 2 - possibly residual tumor, 3 - probably residual tumor, 4 - definitely residual tumor. The readers were not given any instructions or asked to search for certain criteria, and were free to interpreted the scans based on prior experience. Subsequently, both readers were asked to evaluate each of the following imaging criteria using a five-point confidence level score: (a) the presence of an isointense mass (isointense compared with the initial tumor, mostly hyperintense lesions at the time of presentation), (b) the presence of a hypointense (fibrotic) mass, (c) the shape of the tumor (nodular or non-nodular), (d) the aspect of the border of the cervix (regular or irregular), (e) signal homogeneity or heterogeneity, and (f) the shape of the cervix (normal or deformed). The different imaging criteria are shown in Figure 1. All interpretations were performed using MR images obtained after radiotherapy and, if available $(n=29 / 42)$, on MR images obtained immediately before the final BCT application. The pre-treatment images were at the reader's disposal. If MRI was performed immediately before the final $\mathrm{BCT}$, these images were also at the disposal of the reader. The readers were blinded to all other clinical outcomes and imaging.

\section{Standard of reference}

The presence or absence of local residual tumor (in the cervix and/or vagina, parametrium, bladder, and rectum) was determined by: 1) histopathology after surgical resection $(n=6)$; or 2) post-treatment gynecologic examination (under anesthesia) 3 months after the completion of RT, either with $(n=21)$ or without a biopsy (not clinically indicated $n=15$ patients), combined with at least 12 months documented follow-up. Follow-up comprised a gynecologic examination every 3 months, which was conducted by a gynecologic or radiation oncologist. Local residual tumor and/or metastasis was defined as the presence of residual tumor (identified pathologically) or a growing mass on consecutive images, along with an increase in the level of tumor markers in the serum (squamous-cell carcinoma [SCC] or carcinoembryonic antigen [CEA]) [14].

\section{Statistical analysis}

Statistical analyses were performed using SPSS Statistics, v18.0 (SPSS Inc, Chicago, Ill. USA) and Stata v11.0 (StataCorp LP, Texas, USA) software. Interobserver variations were assessed by means of weighted kappa statistics, with quadratic kappa weighting. Each of the MRI criteria were compared and then combined to determine the best combination. Receiver operating characteristics (ROC) curves were constructed to evaluate diagnostic performance for (a) the 'subjective' $\mathrm{T} 2 \mathrm{~W}$ visual response assessment, (b) the 'objective' imaging criteria, and (c) the combined MRI criteria. The areas under the ROC curve (AUC), sensitivity, specificity, and positive- and negative-predictive value were calculated. At the beginning of the study, the decision was made to dichotomize the confidence level scores between 2 and 3 to yield a relatively high sensitivity for residual tumor. The sensitivity and specificity of the different scoring methods were compared using the Mcnemar test (for paired data) or the $\chi^{2}$ test (for unpaired data). The AUCs were compared using to the method described by De Long et al. $P$ values $<0.05$ were considered statistically significant [15].

\section{Results}

\section{Patient and treatment characteristics}

After radiation treatment, seven patients still had local residual tumor; five of these patients also had concurrent distant metastases. Eight patients had distant metastases only, median time to metastases 15 months (range 3-31 months). Twenty-seven patients had no evidence of disease after a median follow-up of 24 months (range 13-81 months). The two patients with local residual tumor but without distant metastasis underwent salvage surgery.

\section{Diagnostic performance for assessment of response}

Figure 2 and Tables 2 and 3 show the diagnostic performance of both readers based on the 'subjective' visual response, the 'objective' imaging criteria, and the combined MRI criteria. The interobserver agreement for the individual imaging criteria is shown in Tables 2, 3, and 4 .

\section{'Subjective' visual assessment after completion of radiotherapy}

For reader 1 (the more experienced), the 'subjective' visual assessment after radiotherapy resulted in an AUC of 0.79 , sensitivity of $71 \%$, and specificity of $86 \%$. For the junior reader (reader 2 ), the results were $0.75,86 \%$, and $46 \%$, respectively. Interobserver agreement for the 'subjective' visual response assessment after treatment was moderate $(\kappa=0.41)$. In 29 patients, additional MR images taken immediately before the final BCT application were available and at the disposal of the reader. Using this MRI, the AUC for reader 1 regarding assessment of the tumor response after completion of RT was 0.99. In the group with 13 patients who did not have this MRI, the AUC was $0.57(p=0.02)$. The corresponding AUCs for reader 2 were 0.90 and $0.60(p=0.08)$.

\section{'Objective' imaging criteria after completion of radiotherapy}

A hypointense mass and signal homogeneity showed a low AUC 0.49-0.63. An isointense mass, a nodular shape, and an irregular border yielded AUCs of 0.79, $0.79-0.83$, and $0.82-0.87$, respectively, when each was used as a stand-alone criterion. Combining all three criteria yielded an AUC of 0.85-0.91. For both readers, using the combined criteria resulted in an AUC higher than that obtained when using 'subjective' visual assessment (AUC 0.91 vs. AUC $0.79, p=0.15$ for reader 1 ; and AUC 0.85 vs. AUC $0.75, p=0.02$ for reader 2). For the less experienced observer, the specificity improved from $46 \%$ to $80 \%$ $(p<0.01)$. Interobserver agreement regarding the individual imaging criteria ranged from $\kappa=0.12$ to $\kappa=0.91$. 

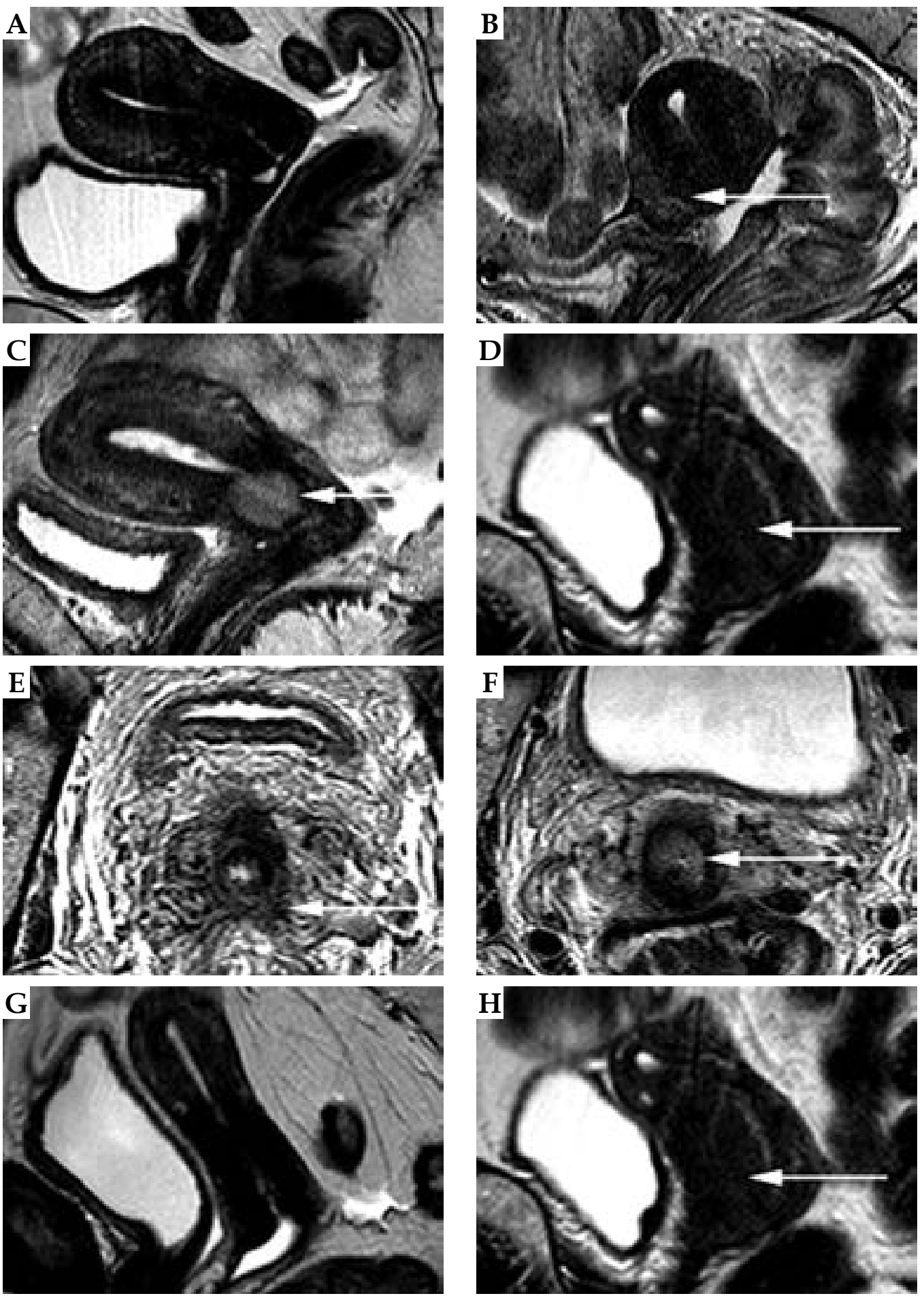

Fig. 1. Sagittal (A-D, G, H) and axial (E, F) T2-weighted images of different patients with a cervical carcinoma after radiotherapy. The patients in A, D, E, G, and $\mathbf{H}$ had no residual tumor. To the contrary: patients in $\mathbf{B}, \mathbf{C}$, and $\mathbf{F}$ showed residual tumor. The images illustrate the imaging criteria used to assess the presence of residual tumour. A, B) The first criteria was the signal intensity of the cervix after treatment, which was scored as hypointense without any signs of an isointense tumour mass (A) or as a persistent isointense signal intensity area, indicative of residual tumour (white arrow in B). C, D) The second criterion was the homogeneity of the signal intensity in the cervix, which was scored as either homogeneous (like the homogeneously isointense lesion indicated by the white arrow in C) or heterogeneous, like the partly isointense and partly hypointense lesion indicated by the arrow in D. E, F) The third and fourth criterion were the aspect of the cervical border and shape (nodular vs. nonnodular) of a (partly) isointense laesion. The cervical border was scored as irregular (E) or regular (F). The shape was scored as either non-nodular (E) or nodular (F). G, H) The final criterion was the presence or absence of persistent deformation of the cervix after treatment. The cervical shape was scored either as normalized $(\mathrm{G})$ or persistently deformed $(\mathbf{H})$ 
The $\kappa$ value for abnormal cervical shape was moderate (0.41). The value for the combined criteria set (isointense mass, a nodular shape, and an irregular border) was 0.84 .

As shown in Table 4 there were no major, nor substantial differences between both observers for the MR criteria set. Both moderate differences correspond to two cases with no evidence of local residual disease after follow-up, in which the less experienced reader scored an isointense mass in contrast to the experienced reader.

\section{Response assessment during brachytherapy}

Magnetic resonance images taken immediately before the final BCT were available for $29 / 42$ patients. The 'subjective' (visual) assessment of MR images taken immediately before the final BCT resulted in an AUC of 0.75 , sensitivity of $75 \%$, and specificity of $36 \%$ for reader 1 , and $0.43,100 \%$, and $8 \%$, respectively, for reader 2 . Each of the three 'objective' imaging criteria on images taken immediately before the final BCT resulted in AUCs of 0.51-0.82. Only abnormal shape and a hypointense mass showed a higher AUC than the subjective assessment when used as stand-alone criteria. Interobserver agreement was rather low (0.29 and 0.46). Only the $\mathrm{K}$ value for isointense mass (0.88) reached an acceptable level.

\section{Discussion}

The present study examined the utility of MRI performed during and after RT (either with or without CT or HT) for detecting residual tumor. We also attempted to identify imaging criteria predictive for residual tumor and assessed their accuracy.

'Subjective' visual assessment of residual disease on post-RT MRI resulted in a reasonable AUC of 0.79 for the more experienced reader and an AUC of 0.75 for the junior reader. The interobserver agreement was poor. However,

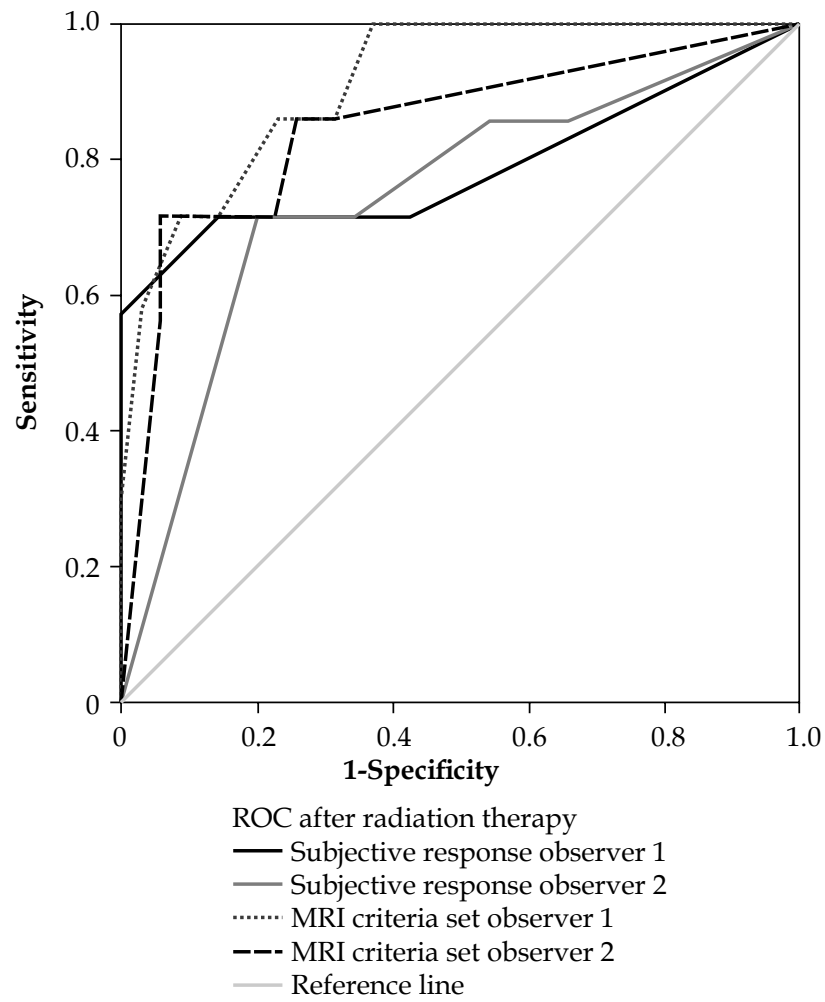

Fig. 2. Reciever operating curve for magnetic resonance imaging (MRI) analysis after radiotherapy, observer 1 and 2

the use of combined 'objective' criteria (isointense mass, nodular shape, and irregular border) to assess residual disease after RT led to a much better performance, and increased the specificity parameter for the less experienced reader. Moreover, the interobserver agreement improved to excellent when the assessment was performed using three 'objective' criteria.

Table 2. Diagnostic performance of 'subjective' and 'objective' magnetic resonance imaging (MRI) criteria analyses for assessing residual tumor $(n=7)$ with MRI after radiotherapy $(n=42)$

\begin{tabular}{|c|c|c|c|c|c|c|c|}
\hline \multirow[t]{3}{*}{ Evaluation } & \multicolumn{2}{|c|}{$\mathrm{AUC}$} & \multirow{3}{*}{$\begin{array}{c}\text { Кappa } \\
\text { interobserver } \\
\text { agreement }\end{array}$} & \multicolumn{4}{|c|}{ Sensitivity and specificity } \\
\hline & \multirow[t]{2}{*}{ Observer 1} & \multirow[t]{2}{*}{ Observer 2} & & \multicolumn{2}{|c|}{ Observer 1} & \multicolumn{2}{|c|}{ Observer 2} \\
\hline & & & & Sensitivity & Specificity & Sensitivity & Specificity \\
\hline $\begin{array}{l}\text { I: 'Subjective' (visual) } \\
\text { evaluation }\end{array}$ & $0.79(0.53-1.00)$ & $0.75(0.53-0.97)$ & $0.41(0.23-0.60)$ & $71 \%(30-94)$ & $86 \%(70-95)$ & $86 \%(42-99)$ & $46 \%(29-63)$ \\
\hline \multicolumn{8}{|c|}{ II: 'Objective' imaging criteria } \\
\hline a. Isointense aspect & $0.79(0.57-1.00)$ & $0.79(0.58-1.00)$ & $0.91(0.81-1.00)$ & $71 \%(30-94)$ & $80 \%(63-91)$ & $71 \%(30-94)$ & $80 \%(63-91)$ \\
\hline b. Hypointense mass* & $0.56(0.31-0.80)$ & $0.63(0.37-0.89)$ & $0.12(0.00-0.38)$ & $14 \%(1-58)$ & $97 \%(83-100)$ & $29 \%(5-70)$ & $97 \%(83-100)$ \\
\hline c. Nodular shape & $0.83(0.63-1.00)$ & $0.79(0.59-0.99)$ & $0.74(0.47-1.00)$ & $71 \%(30-94)$ & $94 \%(79-99)$ & $71 \%(30-94)$ & $83 \%(66-93)$ \\
\hline d. Irregular border & $0.87(0.76-0.98)$ & $0.82(0.65-0.99)$ & $0.78(0.61-0.96)$ & $71 \%(30-95)$ & $77 \%(59-89)$ & $86 \%(42-99)$ & $77 \%(59-89)$ \\
\hline e. Signal homogeneity & $0.61(0.37-0.85)$ & $0.49(0.25-0.72)$ & $0.55(0.22-0.89)$ & $14 \%(1-58)$ & $86 \%(70-95)$ & $14 \%(1-58)$ & $83 \%(66-93)$ \\
\hline f. Abnormal shape & $0.81(0.64-0.99)$ & $0.73(0.47-0.99)$ & $0.41(0.16-0.67)$ & $43 \%(12-80)$ & $89 \%(72-96)$ & $71 \%(30-94)$ & $89 \%(72-96)$ \\
\hline $\begin{array}{l}\text { III: Combined criteria } \\
(a+c+d)\end{array}$ & $0.91(0.80-1.00)$ & $0.85(0.66-1.00)$ & $0.84(0.69-0.99)$ & $71 \%(30-94)$ & $86 \%$ (70-95) & $71 \%(30-94)$ & $80 \%(63-91)$ \\
\hline
\end{tabular}


Table 3. Diagnostic performance of 'subjective' and 'objective' magnetic resonance imaging (MRI) criteria for assessing residual tumor $(n=4)$ with MRI during brachytherapy $(n=29)$

\begin{tabular}{|c|c|c|c|c|c|c|c|}
\hline \multirow[t]{3}{*}{ Evaluation } & \multicolumn{2}{|c|}{ AUC } & \multirow{3}{*}{$\begin{array}{c}\text { Kappa } \\
\text { interobserver } \\
\text { agreement }\end{array}$} & \multicolumn{4}{|c|}{ Sensitivity and specificity } \\
\hline & \multirow[t]{2}{*}{ Observer 1} & \multirow[t]{2}{*}{ Observer 2} & & \multicolumn{2}{|c|}{ Observer 1} & \multicolumn{2}{|c|}{ Observer 2} \\
\hline & & & & Sensitivity & Specificity & Sensitivity & Specificity \\
\hline $\begin{array}{l}\text { I: 'Subjective' (visual) } \\
\text { evaluation }\end{array}$ & $0.75(0.41-1.00)$ & $0.43(0.19-0.66)$ & $0.27(0.02-0.52)$ & $75 \%(22-99)$ & $36 \%(19-57)$ & $100 \%(40-100)$ & $8 \%(1-28)$ \\
\hline \multicolumn{8}{|c|}{ II: 'Objective' imaging criteria } \\
\hline a. Isointense aspect & $0.59(0.34-0.83)$ & $0.54(0.32-0.76)$ & $0.88(0.74-1.00)$ & $75 \%(22-99)$ & $28 \%(13-50)$ & $100 \%(40-100)$ & $24 \%(10-46)$ \\
\hline b. Hypointense mass* & $0.70(0.31-1.00)$ & $0.92(0.00-1.00)$ & $0.46(0.13-0.79)$ & $50 \%(9-91)$ & $84 \%(63-95)$ & $100 \%(40-100)$ & $72 \%(50-87)$ \\
\hline c. Nodular shape & $0.54(0.19-0.88)$ & $0.69(0.40-0.97)$ & $0.37(0.04-0.70)$ & $50 \%(9-91)$ & $52 \%(32-72)$ & $75 \%(22-99)$ & $56 \%(35-75)$ \\
\hline d. Irregular border & $0.55(0.26-0.84)$ & $0.55(0.26-0.84)$ & $0.19(0.00-0.45)$ & $75 \%(22-99)$ & $52 \%(32-72)$ & $100 \%(40-100)$ & $20 \%(8-41)$ \\
\hline e. Signal homogeneity & $0.55(0.18-0.92)$ & $0.51(0.20-0.83)$ & $0.45(0.16-0.73)$ & $50 \%(9-91)$ & $56 \%(35-75)$ & $25 \%(1-78)$ & $76 \%(54-90)$ \\
\hline f. Abnormal shape & $0.82(0.00-1.00)$ & $0.82(0.66-0.98)$ & $0.29(0.00-0.59)$ & $75 \%(22-99)$ & $68 \%(46-84)$ & $100 \%(40-100)$ & $4 \%(0-22)$ \\
\hline
\end{tabular}

$A \cup C$ - area under the receiver operating characteristics curve, ${ }^{*} H y p o i n t e n s e ~ m a s s$, confidence interval $=0$, no hypointense mass, confidence interval $=4$ Sensitivity and specificity are given with the corresponding 95\%

Table 4. Case-by-case comparison and interobserver agreement of 'subjective' and 'objective' magnetic resonance imaging (MRI) for assessing residual tumor after RT $(n=42)$

\begin{tabular}{|c|c|c|c|c|c|c|}
\hline \multirow[t]{3}{*}{ Evaluation } & \multirow{3}{*}{$\begin{array}{c}\text { Interobserver } \\
\text { agreement } \\
\text { Kappa }\end{array}$} & \multicolumn{5}{|c|}{ Observer 1 vs. observer 2} \\
\hline & & \multicolumn{5}{|c|}{$\begin{array}{c}\text { Number of patients }(n) \text { and agreement between observers in these individua } \\
\text { patients }\end{array}$} \\
\hline & & Perfect match $(n)$ & Minor $(n)$ & Moderate $(n)$ & Substantial $(n)$ & Major (n) \\
\hline $\begin{array}{l}\text { I: 'Subjective' (visual) evalu- } \\
\text { ation }\end{array}$ & $0.41(0.23-0.60)$ & 12 & 15 & 8 & 5 & 2 \\
\hline \multicolumn{7}{|l|}{ II: 'Objective' imaging criteria } \\
\hline a. Isointense aspect & $0.91(0.81-1.00)$ & 35 & 4 & 2 & 1 & 0 \\
\hline b. Hypointense mass & $0.12(0.00-0.38)$ & 38 & 1 & 0 & 1 & 2 \\
\hline c. Nodular shape & $0.74(0.47-1.00)$ & 36 & 3 & 0 & 0 & 3 \\
\hline d. Irregular border & $0.78(0.61-0.96)$ & 30 & 7 & 1 & 3 & 1 \\
\hline e. Signal homogeneity & $0.55(0.22-0.89)$ & 34 & 3 & 0 & 1 & 4 \\
\hline f. Abnormal shape & $0.41(0.16-0.67)$ & 22 & 11 & 2 & 5 & 2 \\
\hline $\begin{array}{l}\text { III: Combined criteria } \\
(a+c+d)\end{array}$ & $0.84(0.69-0.99)$ & 34 & 6 & 2 & 0 & 0 \\
\hline
\end{tabular}

Perfect match: no difference in agreement between observers; Minor: 1-point difference, Moderate: 2-point difference, Substantial: 3-point difference, Major: 4-point difference

This is the first report focusing on observer performance when interpreting MRI scans with the aim of identifying residual disease after RT. The results show that using 'objective' criteria improved the performance of both readers. This is due to the introduction of a systematic scoring system, in which certain criteria have to be scored separately. Experienced readers may unconsciously use similar criteria; this may explain why the predictive value for the less expert reader improved to a greater extent than that of the more experienced reader when using the objective criteria rather than the subjective assessment method. However, the experienced reader also improved when using the objective criteria.
The MRI criteria were derived from the literature in conjunction with an independent expert pelvic radiologist. Morphologic visual T2W assessment of cervical tumors is often used to evaluate MR imaging of residual disease after RT [16]. Initial studies reported different values for diagnostic accuracy (sensitivity $80-100 \%$ and specificity 56-100\%) [9,10,11,12]. Vincens et al. reported an equivocal diagnosis in 16/44 cases when iso-/hyperintensity combined with contrast enhancement were used as 'objective' MRI criteria; subjective assessment led to an equivocal diagnosis in only $5 / 44$ cases [12]. However, specificity was still low (55\%) for the group with a definite diagnosis, indicating that isointensity is inaccu- 
rate when used as a single MRI criterion. In our data, we found that isointensity showed a higher specificity ( $80 \%)$. This difference may be explained by the fact that Vincens et al. assessed treatment response at 3-8 weeks after the completion of RT, whereas we assessed the response at a median 9 weeks. Thus, the results of Vincens et al. may have been affected by early radiation effects, with granulomatous tissue formed in the acute post-RT phase mimicking residual tumor isointensity on MRI, resulting in many more false-positives. This hypothesis is supported by the fact that we observed a similarly low specificity for isointensity on MRI performed immediately before the completion of the final BCT.

We found it interesting that both readers performed better when visually assessing the post-RT response of patients for whom an additional scan was performed immediately before the last BCT. The expert reader in particular increased his diagnostic performance. This suggests that the readers found the additional information provided by the BCT MR images very useful during their final assessment of the treatment response. This can be explained by the fact that volume regression over time can be observed more accurately on consecutive scans. This phenomenon was suggested by Mayr et al., who found volume regression analyses after EBRT showed a relatively high diagnostic performance [17].

The prevalence of recurrent/persistent local tumor in our dataset $(17 \%)$ is rather low when compared to that reported in the literature. This may have led to an underestimation of the positive-predictive value. The low recurrence rate may be due to several reasons. First, due to our inclusion criteria, patients who did not finish the imaging or treatment protocols due to the presence of early progressive disease were excluded; $38 \%$ of the current study population had tumors at FIGO stage IIa or less. Second, almost all patients received concurrent CT or HT treatment in addition to RT, which has a higher cure rate than RT alone [18,19]. Third, a substantial proportion of our treatment group received MRI-based BCT. MRI-based $\mathrm{BCT}$ is superior to 2D-based radiotherapy with respect to local relapse rates for cervical carcinomas measuring $>5 \mathrm{~cm}[20,21,22,23]$.

The present study had several limitations. First, the absence of residual disease was not confirmed histopathologically in all cases. This was because not all patients underwent surgery. However, we believe that the absence of recurrence at long-term follow-up is a good indicator of a lack of residual disease. Moreover, when surgery is performed early after RT, microscopic tumors may still be present (RT may not have exerted its full effect at this time). Other research groups tried to solve this problem by including patients with microscopic tumors $(<1 \mathrm{~cm})$ in the complete response group. However, this could lead to an underestimation of the number of patients with recurrent tumor as these foci can still progress to a recurrence. A second limitation is the small number of patients examined. Specifically, the number of patients that underwent MRI during brachytherapy was low; this was because we only started performing MRI-based BCT at our center in 2008. A third limitation was the retrospective nature of the study, which meant that different imaging protocols, different MRI scanners, and different magnetic fields were used. This may have affected the results. However, because $71 \%$ of all post- and pre-treatment scans were performed using a 1.5 Tesla MRI scanner, and all imaging protocols were 'routine clinical protocols', we believe that these differences would not have had a significant effect on the final results. Indeed, the data are representative of those obtained during daily clinical practice.

\section{Conclusions}

The use of predefined 'objective' MRI criteria for assessing residual tumor after RT increases diagnostic performance and makes an accurate diagnosis less dependent upon observer experience. The availability of a brachytherapy MRI scan during treatment improves the detection of residual cervical cancers on post-treatment MRI scans. With the increasing use of MRI during brachytherapy, in future prospective studies, this finding should be investigated more accurately in order to tailor treatment for the patient.

\section{Acknowledgments}

The manuscript was checked for correct English by Bioedit. The assistance was paid by the Gynecologic Oncologic research group of the department of Obstetrics \& Gynecology, Maastricht University Medical Centre, Maastricht, The Netherlands.

There was no funding neither by grands nor external funding by sponsors.

\section{Disclosure}

Authors report no conflict of interest.

\section{References}

1. Kitchener HC, Hoskins W, Small W Jr. et al. The development of priority cervical cancer trials: a Gynecologic Cancer InterGroup report. Int J Gynecol Cancer 2010; 20: 1092-1100.

2. Babar S, Rockall A, Goode A et al. Magnetic resonance imaging appearances of recurrent cervical carcinoma. Int J Gynecol Cancer 2007; 17: 637-645.

3. Elit L, Fyles AW, Devries MC et al. Follow-up for women after treatment for cervical cancer: a systematic review. Gynecol Oncol 2009; 114: 528-535.

4. Nijhuis ER, van der Zee AG, in 't Hout BA et al. Gynecologic examination and cervical biopsies after (chemo) radiation for cervical cancer to identify patients eligible for salvage surgery. Int J Radiat Oncol Biol Phys 2006; 66: 699-705.

5. Keys HM, Bundy BN, Stehman FB et al. Radiation therapy with and without extrafascial hysterectomy for bulky stage IB cervical carcinoma: a randomized trial of the Gynecologic Oncology Group. Gynecol Oncol 2003; 89: 343-353.

6. Hong JH, Tsai CS, Lai $\mathrm{CH}$ et al. Recurrent squamous cell carcinoma of cervix after definitive radiotherapy. Int J Radiat Oncol Biol Phys 2004; 60: 249-257.

7. Houvenaeghel G, Lelievre L, Buttarelli M et al. Contribution of surgery in patients with bulky residual disease after chemoradiation for advanced cervical carcinoma. Eur J Surg Oncol 2007; 33: 498-503.

8. Dresen RC, Beets GL, Rutten HJ et al. Locally advanced rectal cancer: MR imaging for restaging after neoadjuvant radiation therapy with concomitant chemotherapy. Part I. Are we 
able to predict tumor confined to the rectal wall? Radiology 2009; 252: 71-80.

9. Hricak H, Lacey CG, Sandles LG et al. Invasive cervical carcinoma: comparison of MR imaging and surgical findings. Radiology 1988; 166: 623-631.

10. Hatano K, Sekiya Y, Araki H et al. Evaluation of the therapeutic effect of radiotherapy on cervical cancer using magnetic resonance imaging. Int J Radiat Oncol Biol Phys 1999; 45: 639-644.

11. Manfredi R, Maresca G, Smaniotto D et al. Cervical cancer response to neoadjuvant therapy: MR imaging assessment. Radiology 1998; 209: 819-824.

12. Vincens E, Balleyguier C, Rey A et al. Accuracy of magnetic resonance imaging in predicting residual disease in patients treated for stage IB2/II cervical carcinoma with chemoradiation therapy: correlation of radiologic findings with surgicopathologic results. Cancer 2008; 113: 2158-2165.

13. Vliegen RF, Beets GL, Lammering G et al. Mesorectal fascia invasion after neoadjuvant chemotherapy and radiation therapy for locally advanced rectal cancer: accuracy of MR imaging for prediction. Radiology 2008; 246: 454-462.

14. Forni F, Ferrandina G, Deodato F et al. Squamous cell carcinoma antigen in follow-up of cervical cancer treated with radiotherapy: evaluation of cost-effectiveness. Int J Radiat Oncol Biol Phys 2007; 69: 1145-1149.

15. DeLong ER, DeLong DM, Clarke-Pearson DL. Comparing the areas under two or more correlated receiver operating characteristic curves: a nonparametric approach. Biometrics 1988; 44: 837-845.

16. Jeong YY, Kang HK, Chung TW et al. Uterine cervical carcinoma after therapy: CT and MR imaging findings. Radiographics 2003; 23: 969-981; discussion 81.

17. Wang JZ, Mayr NA, Zhang D et al. Sequential magnetic resonance imaging of cervical cancer: the predictive value of absolute tumor volume and regression ratio measured before, during, and after radiation therapy. Cancer 2010; 116: 5093-5101.

18. Chemoradiotherapy for Cervical Cancer Meta-analysis Collaboration (CCCMAC). Reducing uncertainties about the effects of chemoradiotherapy for cervical cancer: individual patient data meta-analysis. Cochrane Database Syst Rev 2010; CD008285.

19. Lutgens L, van der Zee J, Pijls-Johannesma M et al. Combined use of hyperthermia and radiation therapy for treating locally advanced cervix carcinoma. Cochrane Database Syst Rev 2010; CD006377.

20. Pötter R, Dimopoulos J, Georg P et al. Clinical impact of MRI assisted dose volume adaptation and dose escalation in brachytherapy of locally advanced cervix cancer. Radiother Oncol 2007; 83: 148-155.

21. Dimopoulos JC, Petrow P, Tanderup K et al. Recommendations from Gynaecological (GYN) GEC-ESTRO Working Group (IV): Basic principles and parameters for MR imaging within the frame of image based adaptive cervix cancer brachytherapy. Radiother Oncol 2012; 103: 113-122.

22. Pötter R, Georg P, Dimopoulos JC et al. Clinical outcome of protocol based image (MRI) guided adaptive brachytherapy combined with 3D conformal radiotherapy with or without chemotherapy in patients with locally advanced cervical cancer. Radiother Oncol 2011; 100: 116-123.

23. Owrangi AM, Prisciandaro JI, Soliman A et al. Magnetic resonance imaging-guided brachytherapy for cervical cancer: initiating a program. J Contemp Brachytherapy 2015; 7: 417-422. 